\title{
Under diagnosis of intestinal schistosomiasis in a referral hospital, North Ethiopia
}

\author{
Megbaru Alemu ${ }^{1 *}$, Eyob Zigta ${ }^{2}$ and Awoke Derbie ${ }^{1}$
}

\begin{abstract}
Objective: The present cross-sectional study was aimed at determining the magnitude of under diagnosis of intestinal schistosomiasis among patients requested for routine ova/parasite examination at Ayder referral hospital.

Results: A total of 280 stool samples were collected and only $5 \%$ of the patients were positive for ova of Schistosoma mansoni in the routine direct wet mount microscopy. On the other hand, $12.5 \%$ of the patients were positive for ova Schistosoma mansoni when the stool samples were processed by either Kato Kat or formol ether concentration techniques. Moderate test agreement $(k=0.48)$ was recorded for wet mount. Formol-ether concentration $(k=0.89)$ and Kato-Katz ( $\mathrm{k}=0.92$ ) showed excellent agreements with the 'Gold'standard. Direct wet mount technique exhibited the poorest sensitivity (35\%) of detection of ova of Schistosoma mansoni. Hence, the Kato-Katz technique should be implemented in parallel with the direct wet mount microscopy for Schistosoma mansoni presumptive patients.
\end{abstract}

Keywords: Wet mount, Formol ether concentration, Kato-Katz, Mekelle, Ethiopia

\section{Introduction}

Human bilharziasis is caused by the trematode species of Schistosoma mansoni, Schistosoma hematobium, Schistosoma japonicum, Schistosoma intercalatum and Schistosoma mekongi [1]. The disease is highly prevalent throughout Africa, South America and several Caribbean islands [2]. It is one of the most widespread of all human parasitic diseases, ranking second only to malaria in terms of its socioeconomic and public health importance in tropical and subtropical areas [3]. Estimates suggest that over 250 million people were infected and the disease caused 11,700 deaths and a global burden of 3.3 million disability-adjusted life years [4].

In Ethiopia, schistosomiasis is widely spread in the country where endemic areas are located in the altitudinal range of 1200-2000 m above sea level [5]. Rapid spread of the disease also appears to have been facilitated in areas which were originally non-endemic as a result of

\footnotetext{
*Correspondence: mgbeyney@gmail.com

1 Department of Microbiology, Immunology and Parasitology, Bahir Dar University, Po. Box 79, Bahir Dar, Ethiopia

Full list of author information is available at the end of the article
}

the initiation of water-based development schemes [6]. High infection rates of $S$. mansoni were reported in the hyper endemic areas of northwestern, northeastern and northern parts of the country [6-8].

Examination of stool is the primary method of diagnosing suspected Schistosoma mansoni infections. There are several diagnostic techniques such as Kato-Katz, wet-mount, and formol-ether concentration technique (FECT). Despite its low sensitivity, the direct wet mount is the only method employed for diagnostic purpose of intestinal parasites in general and schistosomiasis in particular in health institutions of Ethiopia while FECT and Kato-Katz are reserved for research purpose [9-12]. The Kato-Katz method is characteristically rapid, easy to perform and require minimal training. The formol-ether concentration technique on the other hand is time-consuming, and requires several materials.

Schistosome species lay only few numbers of eggs and only two-third of the eggs are excreted intermittently with stool, making single wet mount microscopy prone for false negative results [13].

The reliable diagnosis of intestinal schistosomiasis therefore requires a more rapid, economical, easy, and

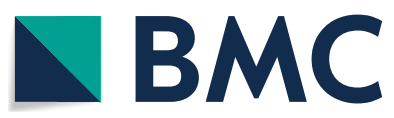

(c) The Author(s) 2018. This article is distributed under the terms of the Creative Commons Attribution 4.0 International License (http://creativecommons.org/licenses/by/4.0/), which permits unrestricted use, distribution, and reproduction in any medium, provided you give appropriate credit to the original author(s) and the source, provide a link to the Creative Commons license, and indicate if changes were made. The Creative Commons Public Domain Dedication waiver (http://creativecommons.org/ publicdomain/zero/1.0/) applies to the data made available in this article, unless otherwise stated. 
sensitive method. Stool examination for intestinal parasitosis is performed solely by wet mount procedure at Ayder referral hospital. It has been a custom that clinicians sending stool specimens of patients with presumptive $S$. mansoni infection to Microbiology and parasitology research laboratory whose stool examination results turned negative in Ayder hospital laboratory. Consequently, ova of S. mansoni were isolated from most of the referred stool specimens in the research laboratory. Hence, we sought to assess performance of wet mount against the Kato-Katz and FECT in the diagnosis of intestinal schistosomiasis and to recommend the best technique in the hospital.

\section{Main text \\ Methods}

\section{Study design and area}

This cross-sectional study was conducted in Ayder referral hospital, North Ethiopia, from August to October 2016. The Hospital provides referral and non-referral services to more than 8 million populations in its catchment areas. It provides a broad range of medical services to both in and outpatients of all age groups. With the total capacity of about 500 inpatient beds in four major departments and other specialty units, the hospital is also used as a teaching hospital for the College of Health Sciences, Mekelle University. Stool examination for intestinal parasitic infections in general and intestinal schistosomiasis in particular is mainly based on direct wet mount microscopy.

\section{Sample collection and parasitological examination}

Patients were provided with stool cups to bring adequate stool samples. The routine direct wet mount microscopy performed in the hospital laboratory. The Kato-Katz and formol-ether concentration (FEC) methods, on the other hand, were performed in microbiology and parasitology research laboratory.

The test procedures were carried out in accordance with standard protocols reported by World Health Organization (WHO) [14].

Kato-Katz method An approximately $42 \mathrm{mg}$ fecal sample was sieved through a $200 \mu \mathrm{m}$ Kato nylon screen mesh. The stool was transferred into a $6 \mathrm{~mm}$ hole of a template on a microscopic slide and covered with glycerol soaked cellophane strip. The microscopic examination then proceeded to identify schistosome eggs and to calculate the number of eggs per gram (EPG) of feces [15]. Based on egg counts, cut-off values for classification of the intensity of infection were used. The intensity of $S$. mansoni was classified into: light infection (1-99 EPG), moderate (100-399 EPG) and heavy ( $\geq 400$ EPG) [14].
Formol ether concentration technique (FEC) Approximately $500 \mathrm{mg}$ of feces was mixed with $10 \mathrm{ml}$ of normal saline and the mixed stool was strained via gauze into a funnel. The strained contents were collected in a centrifuge tube. About $2.5 \mathrm{ml}$ of $10 \%$ formaldehyde (Loba Chemie Pvt Ltd., 107, Wodehouse Road, Jehangir villa, Mumbai-40005, India) and $1 \mathrm{ml}$ of diethyl ether (Blulux laboratories Pvt Ltd. 121005) was then added and centrifuged at $1000 \mathrm{~g}$ for $3 \mathrm{~min}$. The supernatant was removed and a drop of the sediment was covered with cover glass for a microscopic investigation [16].

Wet mount preparation Fresh stool samples (approximately $2 \mathrm{mg}$ of stool) were put on a slide with wooden applicator, emulsified with a drop of physiological saline $(0.85 \%)$ covered with a cover slide and examined at $10 \times$ and $40 \times$ microscopic objectives [16].

\section{Data entry and analysis}

Data were entered and analyzed using SPSS version 20 statistical software. Estimation of the performance of the three diagnostic tests was made by taking the combined results of the wet mount, FEC and Kato-Katz tests as a "Gold" standard diagnostic test, because stool investigation for intestinal parasitosis lacks 'Gold' standard method [17].

Sensitivity, specificity, PPV (positive predictive value), NPV (negative predictive value) and Kappa value of wet mount, FEC and Kato-Katz techniques were computed against the 'Gold' standard. The kappa score was used to estimate the agreement between stool diagnostic tests and the 'Gold' standard.

\section{Data quality control}

Laboratory technicians in charge of microscopic investigations were blinded to the results of the wet mount, FECT, and Kato-Katz. A different laboratory technician was responsible for preparing and reading Kato-Katz and FEC. In addition, results of the wet mount, Kato-Katz, and FEC techniques were recorded on different sheets to ensure strict blinding.

\section{Results}

The overall prevalence of $S$. mansoni infection was 40 (14.3\%) with a parasitic load ranging from 24 to 480 eggs per gram of stool. S. mansoni infections were predominantly light, $65 \%$ and moderate $35 \%$. The peak prevalence of $S$. mansoni infection was recorded for the 10-14 years of age (17.6\%) followed by those 15 and above years of age (14.5\%). Mean intensity of infection was also higher in the age group 10-14 years (224 EPG). The overall prevalence of infection was $12.7 \%$ for females and $15.8 \%$ for male participants $(\mathrm{P}>0.05)$. The mean intensity 
Table 1 Prevalence and intensity of S. mansoni with age and sex at Ayder referral hospital, 2016

\begin{tabular}{lllll}
\hline & \multicolumn{2}{l}{ S. mansoni infection } & Total $\mathbf{n}(\%)$ \\
\cline { 2 - 4 } & Positive $\mathbf{n}$ (\%) & $\begin{array}{l}\text { Negative } \mathbf{n} \\
\text { (\%) }\end{array}$ & $\begin{array}{l}\text { Mean inten- } \\
\text { sity (EPG) }\end{array}$ & \\
\hline Gender & & & & \\
Male & $23(15.8)$ & $123(84.2)$ & 201 & $146(100)$ \\
Female & $17(12.7)$ & $117(87.3)$ & 169 & $134(100)$ \\
Age (years) & & & & \\
$5-9$ & $4(9.3 \%)$ & $39(90.7 \%)$ & 203 & $43(100)$ \\
$10-14$ & $9(17.6)$ & $42(82.4)$ & 224 & $51(100)$ \\
$\geq 15$ & $27(14.5)$ & $159(85.5)$ & 168 & $186(100)$ \\
\hline
\end{tabular}

Table 2 Prevalence of S. mansoni identified in stool diagnostic tests at Ayder referral hospital, 2016

\begin{tabular}{llll}
\hline Diagnostic methods & No examined & Positive $\mathbf{n}(\%)$ & Negative $\mathbf{n}(\%)$ \\
\hline Wet mount & 280 & $14(5.0)$ & $266(95)$ \\
Kato-Katz & 280 & $35(12.5)$ & $245(87.5)$ \\
FEC & 280 & $35(12.5)$ & $245(87.5)$ \\
$\begin{array}{l}\text { Kato-Katz+ wet } \\
\quad \text { mount }\end{array}$ & 280 & $37(13.2)$ & $243(95)$ \\
FEC + wet mount & 280 & $36(12.9)$ & $244(87.1)$ \\
Kato-Katz+FEC & 280 & $38(13.6)$ & $242(86.4)$ \\
KK+FEC + WM & 280 & $40(14.3)$ & $240(85.7)$ \\
\hline
\end{tabular}

of infection was also higher for males (201 EPG) than females (169 EPG) (Table 1).

The prevalence of $S$. mansoni using wet mount, Kato-Katz and FEC was 5, 12.5 and $12.5 \%$, respectively (Table 2). The detection rate when two techniques were used at a time was $13.2 \%$ for wet mount and Kato-Katz, $12.9 \%$ for wet mount and FEC and $13.6 \%$ for Kato-Katz and FEC. The detection rate was $14.3 \%$ when all the three tests were used together (Table 2). Kato-Katz detected 23 samples that were negative by wet mount. Similarly, 21 samples were detected by FEC that were negative by wet mount technique.
The sensitivity of wet mount, Kato-Katz and FEC in the detection of S. mansoni infection was 35 (95\% CI 20.651.7), 87.5 (95\% CI 73.2-95.8), 85 (95\% CI 70.2-94.3), respectively. Similarly, NPV of 90.2 (95\% CI 88-92.1), 98 (95\% CI 95.5-99.1) and 97.6 (95\% CI 95-98.8) were reported, respectively for the wet mount, FEC, and Kato-Katz. Moderate test agreement $(\kappa=0.48)$ was recorded for the wet mount. FEC $(\kappa=0.89)$ and KatoKatz $(\kappa=0.92)$ on the other hand showed excellent agreements with the Gold standard (Table 3).

\section{Discussion}

The prevalence of $S$. mansoni infection in this study was $14.3 \%$, which was higher than previous reports $[18,19]$. This might be explained in part by the nature of the study participants, as symptomatic patients were recruited in our study. The prevalence and intensity of $S$. mansoni were found to be higher in males than females in the current study. This goes in agreement with other studies in Africa [20-22]. The existence of more outdoor activities and water exposure habits among males might have contributed to these findings. The peak prevalence of $S$. mansoni was recorded for the 10-14 age group which was consistent with similar other findings [18].

Diagnosis of intestinal parasitosis is based on detection of the eggs in stool samples examined through a variety of parasitologic methods, and no single technique is satisfactory. In Ethiopia, healthcare laboratories employ the direct wet mount as the preferred stool parasitological examination technique by virtue of its simplicity, low cost, and rapidity.

Although the wet mount technique is the preferred method in resource-limited settings [23], reliance on it as the sole diagnostic tool in routine practice is very likely to cause misdiagnosis of infections. This, in turn, leads to grave clinical and public health consequences [24].

It is supported by our study in which wet mount technique detected only one-third (14/40) of the study participants infected by $S$. mansoni.

The Kato-Katz method is characteristically rapid, easy to perform and require minimal training. Its high sensitivity $(87.5 \%)$ and specificity (100\%) for the diagnosis of

Table 3 The performance of stool diagnostic techniques for diagnosis of intestinal schistosomiasis at Ayder referral hospital, 2016

\begin{tabular}{|c|c|c|c|c|c|}
\hline \multirow[t]{2}{*}{ Diagnostic methods } & \multicolumn{5}{|l|}{ Gold standard } \\
\hline & Sensitivity $(95 \% \mathrm{Cl})$ & Specificity $(95 \% \mathrm{Cl})$ & PPV $(95 \% \mathrm{Cl})$ & NPV $(95 \% \mathrm{Cl})$ & Kappa value \\
\hline Wet mount & $35(20.6-51.7)$ & 100 & 100 & $90.2(88-92.1)$ & 0.48 \\
\hline Kato-Katz & 87.5 (73.2-95.8) & $100(98.5-100)$ & 100 & 98 (95.5-99.1) & 0.92 \\
\hline FEC & 85 (70.2-94.3) & $99.6(97.7-99.9)$ & $97.1(82.7-99.6)$ & $97.6(95-98.8)$ & 0.89 \\
\hline
\end{tabular}


S. mansoni compared to the wet mount is well noted in our study. It was also slightly sensitive than formol-ether concentration. Consistent results were reported by others $[17,25]$. The technique is a useful tool for the quantification of egg counts to determine infection intensities. These qualities make the Kato-Katz the most frequently employed method in research works [14].

Time-consuming procedures, the requirement of trained personnel and several materials/equipment make the FEC technique the most expensive method to be considered as an alternative for routine laboratory diagnosis of intestinal schistosomiasis. However, our study revealed that the sensitivity and specificity of FEC are not much less than the Kato-Katz method for detecting eggs of $S$. mansoni.

The prevalence of $S$. mansonia via wet mount, FEC, and Kato-Katz was 5, 12.5 and $12.5 \%$, respectively. Our study revealed that the Kato-Katz and FEC techniques had about threefold increased in the detection rate of $S$. mansoni than the wet mount. Consistent findings were reported in previous studies [17, 25]. Our study revealed that Kato-Katz (87.5\%) was slightly sensitive than FEC (85\%) to detect S. mansoni. Similarly, Kato-Katz showed the highest agreement with the Gold standard $(\kappa=0.92)$ while wet mount showed the lowest agreement $(\kappa=0.48)$. This showed that the use of Kato-Katz can ultimately reduce misdiagnosis of intestinal schistosomiasis, and reduce morbidity and mortality due to schistosomiasis. This was supported by other findings in Ethiopia [17, 25].

\section{Conclusion}

Direct wet mount technique exhibited the poorest sensitivity of detection of ova of Schistosoma mansoni. Most of the infections were predominantly light in the study area which requires implementation of concentration methods. Hence, the Kato-Katz technique should be implemented in parallel with the direct wet mount microscopy for Schistosoma mansoni presumptive patients.

\section{Limitations of the study}

In this study, we collected only a single stool sample and hence we were unable to describe the variation of egg counts as Schistosoma mansoni female worms undergo intermittent egg excretion. Future investigations should focus on the feasibility of the concentration techniques in terms of the turn-around-time (TAT) in health facilities that serve large number of outpatients.

\section{Abbreviations}

FECT: formol ether concentration techniques; EPG: eggs per gram; WHO: World Health Organization; PPV: positive predictive value; NPV: negative predictive value.
Authors' contributions

MA designed the study and wrote the manuscript; EZ participated in the design of the study, data collection and write-up of the manuscript; AD participated in data analysis and revision of the manuscript. All authors read and approved the final manuscript.

\section{Author details}

${ }^{1}$ Department of Microbiology, Immunology and Parasitology, Bahir Dar University, Po. Box 79, Bahir Dar, Ethiopia. ${ }^{2}$ Department of Medical Parasitology and Vector Biology, Mekelle University, Mekelle, Ethiopia.

\section{Acknowledgements}

We are thankful to medical laboratory staff at Ayder referral hospital.

\section{Competing interests}

The authors declare that they have no competing interests.

\section{Availability of data and materials}

To produce findings of this study, the stated methods and materials were applied. All the data were incorporated in the manuscript and no supplementary files accompanied the submission. The original data supporting this finding will be available at any time upon request.

\section{Consent for publication}

Individual data such as images and videos did not accompany this submission and hence consent for publication is not applicable.

\section{Ethics approval and consent to participate}

Ethical approval was granted by Mekelle University, College of Health Sciences' institutional review board (Reference Number ERC071/2015). Written consents were signed by patients to collect stool specimens for Kato-Katz and FEC processing. Stool samples reported negative by direct wet mount microscopy and turned positive by Kato-Katz and/or FEC were communicated to the hospital to trace patients for possible treatment.

\section{Funding}

The research project was not funded by any organization.

\section{Publisher's Note}

Springer Nature remains neutral with regard to jurisdictional claims in published maps and institutional affiliations.

Received: 5 February 2018 Accepted: 11 April 2018

Published online: 16 April 2018

\section{References}

1. Steinmann P, Keiser J, Bos R, Tanner M, Utzinger J. Schistosomiasis and water resources development: systematic review, meta-analysis and estimates of people at risk. Lancet Infect Dis. 2006;6(4):11-25.

2. Gryseels B, Polman K, Clerinx J, Kestens L. Human schistosomiasis. Lancet. 2006:368:1106-18.

3. Colley DG, Bustinduy AL, Secor WE, King CH. Human schistosomiasis. Lancet. 2014;383(2):253-64.

4. Murray CJL, Vos T, Lozano R, Naghavi M, Flaxman AD, Michuad C, et al. Disability-adjusted life years (DALYs) for 291 diseases and injuries in 21 regions, 1990-2010: a systematic analysis for global burden disease study 2010. Lancet. 2012;380(2):197-223.

5. Erko B, Abebe F, Berhe N, et al. Control of Schistosoma mansoni by the soapberry Endod (Phytolacca dodecandra) in Wollo, North eastern Ethiopia post-intervention prevalence. East Afr Med J. 2002;79:198-201.

6. Tiruneh M, Fantahun M, Kassu A, Tiruneh G, VanLieshout L. Schistosomiasis mansoni in school attenders and non-attenders in Northwest Ethiopia. Ethiop J Health Dev. 2001;15:117-23.

7. Birrie H, Abebe F, Gundersen SG, Medhin G, Berhe N, Gemetchu T. Epidemiology of Schistosomiasis mansoni in three endemic communities in north-east Ethiopia: baseline characteristics before endod based intervention. Ethiop Med J. 1998;36:101-11. 
8. Birrie H, Woldemichael T, Redda A, Chane T. The status of Schistosoma mansoni and snail hosts in Tigray and northern Wello regions, northern Ethiopia. Ethiop Med J. 1994;32:245-54.

9. Utzinger J, Rinaldi L, Lohourignon LK, et al. FLOTAC: a new sensitive technique for the diagnosis of hookworm infections in humans. Trans $R$ Soc Trop Med Hyg. 2008;102(1):84-90.

10. Kassahun $\mathrm{H}$, Abrham D, Yemane $Y$, Birhanu E. Comparision of the Kato katz and FLOTAC techniques for the diagnosis of soil-transmitted helminth infections. Parasitol Int. 2011;60(4):398-402.

11. Knopp S, Rinaldi L, Khamis IS, et al. A single FLOTAC is more sensitive than triplicate Kato-Katz for the diagnosis of low-intensity soil-transmitted helminth infections. Trans R Soc Trop Med Hyg. 2009;103(4):347-54.

12. Tarafder MR, Carabin H, Joseph L, Balolong E, Olveda R, McGarvey ST. Estimating the sensitivity and specificity of Kato-Katz stool examination technique for detection of hookworms, Ascaris /umbricoides and Trichuris trichiura infections in humans in the absence of a 'gold standard'. Int J Para. 2010;40(4):399-404.

13. Gillespie SH, Pearson RD. Principles and practice of clinical parasitology. 2nd ed. Toronto: Wiley; 2001.

14. World Health Organization. Basic laboratory methods in medical parasitology. Geneva: World Health Organization; 1991.

15. World Health Organization. Prevention and control of schistosomiasis and soil-transmitted helminthiasis, vol. 912., WHO technical series reportGeneva: World Health Organization; 2002.

16. Cheesbrough M. District laboratory practice in tropical countries part 1. 2nd ed. New York: Cambridge University Press; 2009.

17. Yimer M, Hailu T, Mulu W, Abera B. Evaluation performance of diagnostic methods of intestinal parasitosis in school-age children in Ethiopia. BMC Res Notes. 2015:8:820.
18. Alemu M, Hailu A, Bugssa G. Prevalence of intestinal schistosomiasis and soil-transmitted helminthiasis among primary school children in Umolante district, South Ethiopia. Clin Med Res. 2014;3(6):174-80.

19. Girum T. The prevalence of intestinal helminthic infections and associated risk factors among school children in Babile town, eastern Ethiopia. Ethiop J Health Dev. 2005;19:140-7.

20. Alembrhan A, Tadesse D, Zewdneh T. Infection prevalence of Schistosoma mansoni and associated risk factors among school children in suburbs of Mekelle city, Tigray, northern Ethiopia. MEJS. 2013;5(1):174-88.

21. Mengistu L, Berhanu E. Prevalence of intestinal parasites among schoolchildren in a rural area close to the southeast of Lake Langano, Ethiopia. Ethiop J Health Dev. 2004;18:116-20.

22. Okpala H, Agwu I, Chimezie O, Nwobu G, Ohihoin A. A survey of the prevalence of schistosomiasis among pupils in Apata and Laranto areas in Jos, Plateau State. OJHAS. 2004;1:1.

23. Wirkom V, Tata R, Agba M, Nwobu G, Ndze R, Onoja O, Utien G, Bongkisheri L, Nsadzetreno V, Banseka E. Formol-petrol stool concentration method: a cheap novel technique for detecting intestinal parasites in resource-limited countries. IJTM. 2007;5(1):1-8.

24. Barnabas MM, Aboi JKM. Missed diagnosis of schistosomiasis leading to unnecessary surgical procedures in Jos university teaching hospital. Trop Dr. 2005;35:96-7.

25. Endris M, Tekeste Z, Lemma W, Kassu A. Comparison of the Kato-Katz, wet mount and formol-ether concentration diagnostic techniques for intestinal helminth infections in Ethiopia. ISRN Parasitol. 2013. https://doi. org/10.5402/2013/180439.
Ready to submit your research? Choose BMC and benefit from:

- fast, convenient online submission

- thorough peer review by experienced researchers in your field

- rapid publication on acceptance

- support for research data, including large and complex data types

- gold Open Access which fosters wider collaboration and increased citations

- maximum visibility for your research: over $100 \mathrm{M}$ website views per year

At $\mathrm{BMC}$, research is always in progress.

Learn more biomedcentral.com/submissions 